Proceeding Series of the Brazilian Society of Computational and Applied Mathematics

\title{
Análise do Modelo Clássico Lotka - Volterra
}

\author{
Anderson Inácio Salata de Abreu ${ }^{1}$ \\ Elenice Weber Stiegelmeier ${ }^{2}$ \\ Michele Cristina Valentino ${ }^{3}$
}

Departamento Acadêmico de Matemática, UTFPR, Cornélio Procópio, PR

Resumo. No presente trabalho será apresentado e investigado um modelo que descreve a interação entre duas espécie do tipo presa - predador, também conhecido como modelo clássico Lotka - Volterra. O objetivo deste trabalho é determinar as soluções e estudar o sistema quanto a sua estabilidade.

Palavras-chave. Modelos populacionais, sistemas de equações, presa - predador.

\section{Introdução}

Os modelos matemáticos de competição e predação são formulados em termos de sistemas não lineares de equações diferenciais ordinárias e tiveram sua origem com os trabalhos de Lotka e Volterra. Dentre os modelo de interação destaca-se o modelo clássico presa predador também conhecido como modelo Lotka - Volterra. O modelo presa - predador trata da interação entre duas espécies onde uma delas dispõe de alimento em abundância (presa) e a segunda espécie (predador) alimenta-se exclusivamente da população de presas $[2]$.

No presente trabalho, será discutido o modelo clássico Lotka - Volterra para duas espécies, o qual descreve um sistema do tipo presa - predador, visando determinar as soluções de equilíbrio e analisa-lo quanto a sua estabilidade.

\section{Modelo Lotka - Volterra}

Seja $x$ e $y$ as populações da presa e do predador, respectivamente, em um instante t. Para a construção do modelo de duas espécie, considere as seguintes hipóteses: i) $\mathrm{Na}$ ausência de predador, a população de presa aumenta exponencialmente a uma taxa proporcional a população atual (modelo de Malthus), ou seja, $d x / d t=a x, a>0$, quando $y=0$. ii) Na ausência da presa, o predador é extinto (morte por falta de alimento), assim, $d y / d t=-b y, b>0$, quando $x=0$. iii) Admitindo que o encontro das duas espécies seja ao

\footnotetext{
1 andersinacio@hotmail.com

${ }^{2}$ elenicew@uftpr.edu.br

3 valentino@utfpr.edu.br
} 
acaso e modelando o número de encontros entre presa e predador como sendo proporcional ao produto das duas populações, ou seja, usando o termo bilinear $x y$.

Considerando as hipóteses apresentadas, o modelo predador-presa é descrito por

$$
\begin{aligned}
& \frac{d x}{d t}=a x-\alpha x y, \\
& \frac{d y}{d t}=-b y+\beta x y
\end{aligned}
$$

com $\alpha$ e $\beta$ representam as medidas de iteração entre as duas espécies. As equações (1) são conhecidas como equações de Lotka - Volterra. Estas equações modelam a iteração entre duas espécies, onde a primeira (presa) dispõe de alimento em abundância e a segunda espécie (predador) alimenta-se da primeira.

Os pontos críticos do sistema (1) são as soluções das equações algébricas

$$
\begin{aligned}
x(a-\alpha y) & =0 \\
y(-b+\beta x) & =0 .
\end{aligned}
$$

Nesse caso, os pontos críticos são $p_{1}=(0,0)$ e $p_{2}=\left(\frac{b}{\beta}, \frac{a}{\alpha}\right)$.

Analisando o comportamento local das soluções perto de cada ponto crítico, a partir do sistema linear correspondente, pode-se concluir que a origem, $p_{1}$, é um ponto de sela e, portanto, instável, ou seja, todos as trajetórias se afastam de uma vizinhança da origem. O ponto crítico $p_{2}$ é um centro estável, assim, as trajetórias na primeiro quadrante circulam em torno do ponto crítico $p_{2}$. Essa análise pode ser feita com o auxílio do retrato de fase do sistema (1) ou do campo de direções [3].

Para vários sistemas do tipo predador-presa o modelo de Lotka - Volterra não pode ser aplicado devido as suas limitações, ou seja, descreve apenas oscilações periódicas, para corrigir este problema foram formulados outros problemas mais ricos e realístico [2].

\section{Considerações finais}

Nesse trabalho foi apresentado o modelo clássico de Lotka - Volterra para a interação entre duas espécies, bem como, a análise de estabilidade. Posteriormente, pretende-se estudar a aplicação de modelos do tipo presa-preador no controle biológico de populações.

\section{Referências}

[1] W. E. Boyce, R.C. Diprima, Equações Diferenciais Elementares e Problemas de Valores de Contorno, Editora LTC, São Paulo, (1994).

[2] L. Edelstein-Keshet, Mathematical Models in Biology, Random House, New York, (1988).

[3] D. G. Figueiredo, A. F. Neves, Equações Diferenciais Aplicadas, 3 ed., IMPA, Rio de Janeiro, (2014). 